\title{
Aparelhos de amplificação sonora individual: quais são as queixas mais recorrentes dos usuários e suas possíveis relações com ajustes finos?
}

\author{
Hearing aids: what are the most recurrent complaints from users and \\ their possible relationship with fine tuning?
}

Laura Franco Chiriboga ${ }^{1}$ (D), Christiane Marques do Couto ${ }^{2}$ (i), Kátia de Almeida ${ }^{3}$ (1)

\section{RESUMO}

Objetivo: identificar as queixas mais recorrentes dos usuários de aparelhos de amplificação sonora individual (AASI) e estabelecer possíveis relações que auxiliem a busca de soluções de problemas no processo de adaptação desses dispositivos. Métodos: estudo transversal, descritivo e quantitativo, realizado mediante questionário on-line, respondido por 176 fonoaudiólogos. Após computação e organização das respostas, realizou-se uma codificação e foram descritos 30 termos mais recorrentes, referentes às queixas dos usuários. Foram estabelecidas quatro categorias para verificar cada termo relatado e sua correspondência com o problema apontado em cada questão. Para o direcionamento das possíveis relações entre as queixas e suas resoluções, os termos foram divididos nos seguintes aspectos principais do processo de adaptação de AASI: adaptação física, ajuste das características eletroacústicas e características intrínsecas do usuário. Resultados: foram descritos 30 termos representativos das queixas, com maior número de ocorrências para o termo "Aparelho muito alto/Som muito alto", que apareceu 223 vezes. O termo "Não escuta nada" apareceu com menor número de ocorrências, 25 vezes. No total, foram encontradas seis queixas relacionadas a aspectos físicos, 17 a ajustes de características eletroacústicas, quatro que poderiam estar relacionadas a ambos os aspectos e duas a características intrínsecas do indivíduo. Conclusão: foi possível elencar as queixas mais referidas pelos usuários de AASI. Observou-se que é viável, a partir de uma queixa, direcionar um suposto aspecto relacionado à adaptação e auxiliar os fonoaudiólogos a levantar soluções, apesar de ser clara a necessidade de um olhar individualizado para cada paciente e situação.

Palavras-chave: Audição; Perda auditiva; Auxiliares de audição; Audiologia; Resolução de problemas

\begin{abstract}
Purpose: To identify the most frequent complaints from hearing-aid users and establish possible relationships that might help audiologists solve some problems. Methods: Cross-sectional, descriptive and quantitative study, carried out through an online questionnaire, answered by 176 audiologists. After analyzing the responses and computing the terms, coding was performed and the 30 most frequent complaints were described. Four categories were established to verify each reported term and its correspondence with the problem pointed out in each question. To address the possible relationships between complaints and their solutions, the terms were divided into the following main aspects of the hearing aid fitting process: physical adaptation, adjustment of electroacoustic characteristics and user's intrinsic characteristics. Results: 30 representative terms of complaint were described due to their high number of occurrences : the term "very loud device/ very loud sound" appeared 223 times whereas the term "I cannot hear anything" had the fewest number of occurrences, 25 appearances. In total, there were six complaints related to physical aspects, 17 to adjustments of electroacoustic characteristics, four that could be related to both aspects and two to intrinsic characteristics of the individual. Conclusion: It was possible to identify the most frequently reported complaints of hearing aid users. . It was observed that it is feasible, based on a complaint, to address a supposedly related aspect of adaptation and help audiologists find solutions. However, each particular patient and situation must be given individualized attention.
\end{abstract}

Keywords: Hearing; Hearing loss; Hearing aids; Audiology; Problem solving

\footnotetext{
Trabalho realizado na Universidade Estadual de Campinas - UNICAMP - Campinas (SP), Brasil.

${ }^{1}$ Programa de Pós-graduação em Saúde, Interdisciplinaridade e Reabilitação, Departamento de Desenvolvimento Humano e Reabilitação, Faculdade de Ciências Médicas, Universidade Estadual de Campinas - UNICAMP - Campinas (SP), Brasil.

${ }^{2}$ Departamento de Desenvolvimento Humano e Reabilitação, Faculdade de Ciências Médicas, Universidade Estadual de Campinas - UNICAMP - Campinas (SP), Brasil.

${ }^{3}$ Faculdade de Ciências Médicas, Santa Casa de São Paulo - FCMSCSP - São Paulo (SP), Brasil.

Conflito de interesses: Não.

Contribuição dos autores: LFC participou da idealização do estudo, coleta, análise e interpretação dos dados e redação do artigo; CMC participou, na condição de orientadora, da idealização do estudo e revisão do artigo; KA participou da revisão do artigo.

Financiamento: Nada a declarar.

Autor correspondente: Laura Franco Chiriboga. E-mail: laura.chiriboga@hotmail.com

Recebido: Julho 21, 2021; Aceito: Dezembro 21, 2021
} 


\section{INTRODUÇÃO}

De acordo com projeções realizadas pela Organização Mundial da Saúde (OMS), até 2050, uma em cada dez pessoas terá perda auditiva (PA) incapacitante ${ }^{(1)}$. Portanto, sabe-se que a PA é um tema cada vez mais discutido e presente no cenário mundial, já que, independentemente da idade, traz consequências negativas na comunicação, no estado emocional e na qualidade de vida ${ }^{(2)}$. A amplificação sonora é a intervenção mais recomendada para indivíduos com PA e, dentre os dispositivos tecnológicos disponíveis, os aparelhos de amplificação sonora individual (AASI) são vastamente utilizados ${ }^{(3)}$ e apresentam benefícios comprovados $^{(4-6)}$. Para garantir uma adaptação de excelência, é preciso seguir as etapas de boas práticas preconizadas pela Academia Americana de Audiologia ${ }^{(7)}$ e pela Academia Brasileira de Audiologia ${ }^{(8)}$. São elas: avaliação do indivíduo, aspectos técnicos do tratamento, orientação e aconselhamento do usuário e validação do uso da amplificação.

No entanto, ainda falta padronização e protocolo para ajustes finos, principalmente quanto aos demais recursos oferecidos pelos AASI, além daqueles relacionados ao ajuste do ganho por frequência ${ }^{(9)}$. Para auxiliar a prática atual, estudos sugerem, baseados em evidências, o desenvolvimento de ferramentas que contribuem para a resolução das queixas, levando também em consideração características individuais do usuário ${ }^{(9,10)}$. Desse modo, reforça-se que, no processo de adaptação do AASI, é necessária a participação ativa do usuário. Ouvir o que ele tem a dizer sobre sua audição amplificada é tarefa fundamental para individualizar o atendimento, respeitar suas características e valorizar suas queixas ${ }^{(11)}$. A escuta ocorre durante todo o processo de adaptação e a sensibilidade do fonoaudiólogo para entender o que representa cada queixa do paciente e como trabalhar com ela é indispensável para a resolução dos problemas apresentados.

Com a necessidade de se entender os termos utilizados pelos usuários de AASI e como tais descrições poderiam contribuir para o processo de adaptação, foram realizados diversos estudos, na década de 1990, a respeito de descritores de sons e de ajustes em AASI, principalmente na Suécia ${ }^{(12-14)}$. O principal objetivo dos estudos era analisar se os usuários de AASI seriam capazes de descrever o som de maneira precisa, para que essas descrições fossem utilizadas para os ajustes finos dos dispositivos. Constatou-se que é possível que os usuários descrevam com precisão suas percepções para o uso nos ajustes de seus AASI. Outras pesquisas relacionaram as queixas dos usuários de AASI a parâmetros de ajustes específicos, também com resultados de fidedignidade entre os relatos e os ajustes finos $^{(15-17)}$.

O estudo de Jenstad et al. ${ }^{(17)}$ teve o objetivo de levantar os termos mais utilizados por usuários de AASI para descrever suas queixas e, assim, elaborar um guia de ajustes finos baseado nessas queixas. Foram levantados 40 descritores mais frequentes sobre comportamento eletroacústico e sobre características físicas dos AASI. Os autores relacionaram esses descritores com futuros ajustes que poderiam guiar os audiologistas nos ajustes finos. Outra pesquisa ${ }^{(18)}$ foi realizada na Holanda, com a intenção de replicar o estudo citado anteriormente, porém, para a realidade da língua holandesa. Os estudos referidos foram realizados na língua inglesa e holandesa e demonstram que os termos e resultados encontrados não podem extrapolar o uso para outras línguas, pois cada língua apresentar particularidades, variações e origens distintas.
Na literatura consultada, não foram encontradas pesquisas recentes, nacionais ou internacionais, que descrevam termos utilizados para as queixas espontâneas mais frequentes dos usuários de AASI e possíveis resoluções dos problemas. Por conta disso, percebeu-se que era necessária a descrição dos termos em português brasileiro, visando ao entendimento maior de quais são as queixas referidas pelos usuários de AASI.

Constatou-se, além disso, a necessidade de compreender de que modo o fonoaudiólogo deve olhar para esses aspectos, pensando nas características físicas e eletroacústicas dos AASI, com intuito de atingir os objetivos propostos pela sua adaptação e pela reabilitação auditiva. Dessa forma, esta pesquisa teve como objetivo identificar as queixas mais recorrentes dos usuários de aparelhos de amplificação sonora individual e estabelecer possíveis relações que auxiliem a solução de problemas no processo de adaptação desses dispositivos.

\section{MÉTODOS}

Trata-se de um estudo transversal, descritivo e quantitativo. O projeto foi encaminhado ao Comitê de Ética em Pesquisa - CEP da FCM/UNICAMP e aprovado sob o número 2.253.925/2017. A coleta de dados foi iniciada somente após a aprovação do CEP.

\section{Procedimentos}

A pesquisa foi realizada e viabilizada por meio de um questionário on-line, elaborado na plataforma Google Forms, baseada no estudo de Jenstad et al. ${ }^{(17)}$ e divulgada por redes sociais e e-mails. Não houve divulgação por meio de associações profissionais ou conselhos de classe. Os sujeitos da pesquisa declararam sua anuência ao clicar no botão "aceito participar", existente no final do Termo de Consentimento Livre e Esclarecido (TCLE) on-line. Somente após a anuência do TCLE os fonoaudiólogos tiveram acesso ao questionário.

O questionário foi dividido em duas partes, em que a primeira foi composta por 11 questões a respeito dos participantes e de seus locais de trabalho. A segunda parte continha 18 questões abertas, subdivididas em cinco categorias principais que influenciam na adaptação de AASI: ganho por frequência, volume/saída máxima, adaptação física, características de compressão e sons indesejáveis (Quadro 1). Todas as questões foram delineadas de maneira a permitir que o fonoaudiólogo relatasse a queixa do paciente quando o AASI está se comportando de determinada maneira.

Após a análise e categorização das queixas apresentadas, as possíveis relações entre essas queixas e soluções de problemas no processo de adaptação desses dispositivos foram levantadas pelos pesquisadores responsáveis, durante a condução do estudo. Para o estabelecimento dessas relações, os termos foram dimensionados nos seguintes aspectos principais: adaptação física, ajuste das características eletroacústicas e características intrínsecas do usuário. É importante considerar que: adaptação física está relacionada às características físicas do AASI e também às condições anatômicas do paciente; ajuste das características eletroacústicas pressupõe a realização de ajustes finos e regulagem no dispositivo; características intrínsecas do usuário se referem a fatores inerentes ao indivíduo, tais como 
Quadro 1. Questionário

Ao fonoaudiólogo que está respondendo
este questionário, informamos que todas
as questões devem ser lidas contendo
a seguinte frase após cada item: “meus
pacientes estão acostumados a usar os
termos ou frases".
Exemplo: "Se a adaptação tem muito
ganho nas baixas frequências, meus
pacientes estão acostumados a usar os
termos ou frases: 'o aparelho está meio
grosso' ou 'o aparelho está dando eco".'
Deve ser colocada a frase/palavra que
o paciente usa para se expressar sobre
o aparelho quando ele está ajustado/se
comportando de determinada maneira.
Não é necessário que o fonoaudiólogo
escreva em sua resposta a frase "meus
pacientes estão acostumados a usar os
termos ou frases", somente colocar as
queixas que os pacientes referem.

Ganho por frequência

Se a adaptação tem muito ganho nas baixas frequências...

Se a adaptação tem muito ganho nas altas frequências...

Se a adaptação tem muito ganho nas médias frequências..

\section{Volume/Saída Máxima}

Se a adaptação tem muito ganho geral... Se a adaptação tem pouco ganho geral... Se a saída máxima é muito alta... Se a saída máxima é muito baixa...

\section{Adaptação Física}

Se o paciente relata oclusão...

Se o molde ou oliva está muito apertado... Se o molde ou oliva está muito largo...

Legenda: $\mathrm{AASI}$ = aparelho de amplificação sonora individual

capacidade cognitiva e funcionamento do cérebro e funções executivas superiores para compreensão de fala.

\section{Amostra}

A amostra foi composta por 176 fonoaudiólogos que seguiram os seguintes critérios de inclusão: profissionais com graduação em Fonoaudiologia no Brasil e profissionais que atuam ou atuaram na área de aparelhos de amplificação sonora individual. Não foi considerada relevante para a análise das respostas a diferenciação entre profissionais que estavam atuando no momento ou que já haviam atuado, visto que os fonoaudiólogos poderiam desistir de responder ao questionário caso não se sentissem à vontade ou gabaritados. Foram excluídos os questionários cujas respostas fugiram do escopo da pesquisa, como, por exemplo, respostas de "sim" ou "não" quando era solicitado descrever termos ou frases que os usuários de AASI utilizavam.

Desses profissionais, 93\% (163) eram do sexo feminino e $7 \%$ (13) eram do sexo masculino; $70 \%$ dos fonoaudiólogos eram da Região Sudeste, 14\% da Região Sul, 7\% do Nordeste, 3\% da Região Norte, $3 \%$ da Região Centro-Oeste e 3\% não informaram esse dado. Em relação à formação, 31 fonoaudiólogos tinham apenas graduação, 94, pós-graduação lato sensu, 28 eram mestres e 23 tinham título de doutorado. A média de tempo de experiência com adaptação de AASI foi de 10,81 anos, sendo que o mínimo foi de seis meses e o máximo, de 40 anos.

\section{Análise estatística}

A análise estatística inferencial dos dados se deu a partir dos seguintes passos: 1) Codificação dos termos: feita por duas profissionais fonoaudiólogas responsáveis pela pesquisa, com experiência mínima de três anos e especialização na área de seleção e adaptação de AASI. Antes de sua realização, houve uma preparação por meio do estudo dos termos existentes e revisão de regras da língua portuguesa. Após, a codificação se deu de forma independente. Quando ocorreu divergência, procurou-se conversar e entrar em consenso quanto à classificação. Os termos com o mesmo significado (como, por exemplo: "tem um barulho no meu aparelho" e "meu aparelho está fazendo um barulho") e com o mesmo morfema ("eco", "ecoa", "ecoando") foram combinados em uma mesma categoria. Os morfemas que modificavam o sentido da palavra não foram agrupados na mesma categoria. Apesar de existirem softwares específicos para mineração de dados de texto do estudo, eles não se mostraram úteis em termos de língua portuguesa. Foi necessária a seleção manual a partir dos dados tabulados no editor de planilhas Microsoft Office Excel; 2) Cálculo da frequência da ocorrência para cada termo em relação ao questionário inteiro e verificação dos termos mais frequentes (neste estudo, foram relacionados os 30 termos mais frequentes); 3) Verificação dos termos que se repetem nas diferentes questões. Isso fez com que fosse possível analisar cada termo e quais os prováveis comportamentos de processamento sonoro e características físicas que os AASI podem apresentar. Para tanto, foi realizada uma análise da distribuição dos termos por questões e da quantidade de vezes que esses termos foram referidos nas questões. Da tabela de frequência da ocorrência dos termos, foram selecionados $10 \%$ do total dos termos mais frequentes, ou seja, os 30 primeiros itens que estavam apresentados em ordem decrescente (do maior número de ocorrências de cada termo para o menor número). Para a análise dos resultados, foram estabelecidas quatro categorias: A, B, C e D (Tabela 1)

\section{RESULTADOS}

A Tabela 2 apresenta a ocorrência dos 30 termos referentes a queixas de usuários de AASI em ordem decrescente, ou seja, do termo que apresentou maior número de ocorrências para o termo que apresentou menor número de ocorrências. Observou-se que o termo mais frequente, "Aparelho muito alto/Som muito alto", apareceu 223 vezes e o termo menos frequente, "Não ouve nada", apareceu 25 vezes.

O Quadro 2 demonstra como as categorias definidas (seção Métodos) foram analisadas e descritas. Foi possível observar 
que a maioria dos termos obtidos apareceram em mais de uma questão, mas de maneira não homogênea, ou seja, o seu aparecimento prevaleceu em determinadas questões. A partir dessa categorização, foram apresentadas as supostas relações que podem auxiliar a solução de problemas no processo de adaptação dos AASI.

\section{DISCUSSÃO}

A análise das respostas da pesquisa permitiu conhecer os termos mais referidos por usuários de AASI para descrever suas queixas, relatadas pelos fonoaudiólogos que os acompanhavam. Foram observados 30 termos de maior ocorrência (Tabela 2) para categorização e descrição das queixas dos usuários. Os outros estudos que realizaram o levantamento desses termos na língua inglesa $^{(17)}$ e holandesa ${ }^{(18)}$ elaboraram uma lista de 40 vocábulos mais frequentes relatados por usuários de AASI. Os termos do estudo holandês estão descritos em inglês por conta de razões de publicação, como relata o autor ${ }^{(18)}$. Não foi encontrado nenhum estudo semelhante em língua portuguesa. A Tabela 3 faz uma comparação dos dez primeiros termos entre as três pesquisas.

O termo mais referido no presente estudo foi "Aparelho muito alto/Som muito alto", dado que concorda com os achados dos termos semelhantes aos estudos de língua inglesa e holandesa. Ao analisar os dez descritores das outras pesquisas ${ }^{(17,18)}$, relacionando-as com a realizada no Brasil, observou-se que, apesar das línguas distintas e do longo tempo de realização do

Tabela 1. Categorias de análise e seus parâmetros para a distribuição dos termos por questões

\begin{tabular}{|c|c|}
\hline Categorias & Parâmetros \\
\hline Categoria A: O termo só apareceu em uma questão. & $100 \%$ das vezes o termo apareceu em determinada questão. \\
\hline $\begin{array}{l}\text { Categoria B: O termo apareceu em mais de uma questão, porém } \\
\text { com maior ocorrência em uma delas. }\end{array}$ & $\begin{array}{l}\text { Maior valor de ocorrência do termo representou } \geq 50 \% \text { do total de } \\
\text { vezes que o termo foi apresentado. }\end{array}$ \\
\hline $\begin{array}{l}\text { Categoria C: O termo apareceu em mais de uma questão, sendo que } \\
\text { uma delas se destacava, porém outras também apresentavam valores } \\
\text { relevantes. }\end{array}$ & $\begin{array}{l}\text { Soma dos dois ou três maiores valores da ocorrência dos termos } \\
\text { representou } \geq 50 \% \text { do total de vezes que os termos foram } \\
\text { apresentados. }\end{array}$ \\
\hline $\begin{array}{l}\text { Categoria D: O termo apareceu em mais de uma questão e foi } \\
\text { distribuído homogeneamente em todas as questões em que apareceu. }\end{array}$ & $\begin{array}{l}\text { Soma dos três maiores valores da ocorrência dos termos representou } \\
\leq 50 \% \text { do total de vezes que os termos foram apresentados. }\end{array}$ \\
\hline
\end{tabular}

Tabela 2. Ocorrência de termos referentes a queixas de pacientes conforme relatado pela amostra do estudo

\begin{tabular}{|c|c|c|}
\hline$\#$ & Termo & $\mathrm{N}^{\circ}$ de ocorrências \\
\hline 1 & Aparelho muito alto/Som muito alto & 223 \\
\hline 2 & Aparelho apitando & 222 \\
\hline 3 & Aparelho abafado/Som abafado & 180 \\
\hline 5 & Som estridente & 136 \\
\hline 6 & Aparelho baixo/Som baixo & 132 \\
\hline 9 & Aparelho chiando/Som chiado & 97 \\
\hline 10 & Aparelho incomoda/Som incomoda/incômodo & 97 \\
\hline 11 & Dificuldade para interpretação da fala & 92 \\
\hline 12 & Machuca (aparelho) & 72 \\
\hline 13 & Aparelho fraco/Som fraco & 72 \\
\hline 14 & Ouvido tampado/Ouvido tampado (aparelho) & 69 \\
\hline 18 & Som distorcido & 54 \\
\hline 19 & Aparelho não faz diferença/Não faz diferença & 50 \\
\hline 20 & Som cortando/Fala cortada & 48 \\
\hline 21 & Aparelho sai & 43 \\
\hline 22 & Incômodo físico & 42 \\
\hline 23 & Som forte incomoda & 40 \\
\hline 24 & Som metálico & 40 \\
\hline 25 & Própria voz dentro da cabeça & 36 \\
\hline 26 & Aparelho ruidoso/Ruído/Muito ruído & 35 \\
\hline 27 & Própria voz alta & 30 \\
\hline 28 & Não ouve bem & 29 \\
\hline
\end{tabular}


estudo da língua inglesa (mais de uma década), os termos usados para descrever o comportamento do AASI são semelhantes.

Ao longo do tempo, os fabricantes de AASI demonstraram grande empenho no desenvolvimento de tecnologias para aprimorar o processamento sonoro, as quais foram se atualizando de forma exponencial. O desenvolvimento de novos algoritmos, assim como atualizações das regras prescritivas, estão presentes a todo momento, com intuito de amenizar as queixas e melhorar o desempenho dos pacientes com esses dispositivo eletrônicos ${ }^{(19)}$. Desse modo, o ajuste fino dos AASI se torna cada vez mais complexo em razão dos avanços tecnológicos e pelo fato de que os aparelhos atuais tentam compensar não somente a magnitude (grau) da perda auditiva, como também a maneira de processar o som, ao “imitar” a cóclea, por meio dos métodos de amplificação não linear e ativação de diferentes estratégias de processamentos sonoros para distintos ambientes acústicos ${ }^{(20-22)}$. Apesar disso, observa-se que as queixas permanecem semelhantes, mesmo após mais de uma década de diferença entre os estudos americano, holandês e brasileiro. Isso reforça que, mesmo com os avanços, algumas dificuldades apresentadas pelos usuários de AASI ainda não foram passíveis de solução.

Torna-se evidente que, embora com grandes avanços, os AASI não conseguem resgatar o processamento sonoro realizado por um sistema auditivo íntegro. Contudo, para as modificações possíveis necessita-se de um fonoaudiólogo capacitado a manejar a programação do AASI.

Esse levantamento de termos representativos das prováveis queixas mais frequentes possibilita que o profissional as analise e proponha caminhos para realizar ajustes finos e orientações para auxiliá-lo em uma adaptação mais eficiente e um processo de reabilitação auditiva adequado. Assim como em outras pesquisas, demonstrou-se que existe uma diversidade de queixas e de dificuldades relacionadas ao uso dos AASI, reforçando o desafio contínuo que os usuários e, também, os fonoaudiólogos

Tabela 3. Descrição dos dez primeiros termos nos três estudos: português brasileiro, inglês e holandês

\begin{tabular}{|c|c|c|c|}
\hline Termos & $\begin{array}{c}\text { Esta Pesquisa } \\
\text { Língua portuguesa }\end{array}$ & $\begin{array}{c}\text { Jenstad et al. } \\
\text { (17) Língua } \\
\text { inglesa } \\
\end{array}$ & $\begin{array}{c}\text { Thielemans et al. } \\
\text { (18) Língua } \\
\text { holandesa } \\
\end{array}$ \\
\hline 1. & $\begin{array}{l}\text { Aparelho muito alto/ } \\
\text { Som muito alto }\end{array}$ & Too loud * & Too loud \\
\hline 2. & Aparelho apitando & Whistle & Too sharp \\
\hline 3. & $\begin{array}{l}\text { Aparelho abafado/ } \\
\text { Som abafado }\end{array}$ & Not clear & $\begin{array}{l}\text { Bad speech } \\
\text { understanding }\end{array}$ \\
\hline 4. & $\begin{array}{l}\text { Aparelho com eco/ } \\
\text { Som com eco/Eco na } \\
\text { cabeça/Própria voz } \\
\text { com eco }\end{array}$ & Pain & Dull \\
\hline 5. & Som estridente & $\begin{array}{l}\text { In a barrel, } \\
\text { tunnel, or well }\end{array}$ & Too soft \\
\hline 6. & $\begin{array}{l}\text { Aparelho baixo/Som } \\
\text { baixo }\end{array}$ & Tinny & Indistinct \\
\hline 7. & $\begin{array}{l}\text { Aparelho muito forte/ } \\
\text { Som muito forte }\end{array}$ & Muffled & $\begin{array}{l}\text { Hearing aid } \\
\text { whistles }\end{array}$ \\
\hline 8 & $\begin{array}{l}\text { Aparelho faz barulho/ } \\
\text { Muito barulho }\end{array}$ & $\begin{array}{l}\text { Environmental } \\
\text { sounds too } \\
\text { loud }\end{array}$ & Distorted \\
\hline 9. & $\begin{array}{l}\text { Aparelho chiando/Som } \\
\text { chiado }\end{array}$ & $\begin{array}{l}\text { Not loud } \\
\text { enough }\end{array}$ & $\begin{array}{l}\text { Hearing aid is } \\
\text { producing noise }\end{array}$ \\
\hline 10. & $\begin{array}{l}\text { Aparelho incomoda/ } \\
\text { Som incomoda/ } \\
\text { Incômodo }\end{array}$ & Sharp & Too shrill \\
\hline
\end{tabular}

Quadro 2. Resultado da análise das quatro categorias propostas e suas relações com a adaptação do aparelho de amplificação sonora individual

\begin{tabular}{|c|c|c|c|}
\hline Categoria & Parâmetros & Análise & Possíveis relações \\
\hline Categoria A & $100 \%$ & $\begin{array}{l}3 \text { termos: Aparelho sai, Incômodo físico, Aparelho } \\
\text { escapa. }\end{array}$ & $\begin{array}{l}\text { Adaptação física: Aparelho sai, Incômodo físico, } \\
\text { Aparelho escapa. }\end{array}$ \\
\hline \multirow[t]{2}{*}{ Categoria B } & \multirow[t]{2}{*}{$\begin{array}{l}\text { Maior valor } \geq 50 \% \\
\text { do total }\end{array}$} & \multirow[t]{2}{*}{$\begin{array}{l}12 \text { termos: Aparelho apitando, Som estridente, } \\
\text { Aparelho baixo/Som baixo, Machuca (aparelho), } \\
\text { Ouvido tampado/Ouvido tampado (aparelho), } \\
\text { Dor física/Dor de orelha, Aparelho estranho/Som } \\
\text { estranho, Aparelho não faz diferença/Não faz } \\
\text { diferença, Som forte incomoda, Som metálico, } \\
\text { Própria voz dentro da cabeça, Não ouve nada. }\end{array}$} & $\begin{array}{l}\text { Adaptação física: Machuca (aparelho), Ouvido } \\
\text { tampado/Ouvido tampado (aparelho), Dor física/Dor de } \\
\text { orelha. } \\
\text { Ajuste das características eletroacústicas: Som } \\
\text { estridente, Aparelho estranho/Som estranho, Aparelho } \\
\text { não faz diferença/Não faz diferença, Som forte } \\
\text { incomoda, Som metálico, Não ouve nada. }\end{array}$ \\
\hline & & & $\begin{array}{l}\text { Adaptação física ou ajuste das características } \\
\text { eletroacústicas: Aparelho apitando, Própria voz dentro } \\
\text { da cabeça. }\end{array}$ \\
\hline \multirow[t]{3}{*}{ Categoria C } & \multirow[t]{3}{*}{$\begin{array}{l}\text { Soma dos dois } \\
\text { ou três maiores } \\
\text { valores } \geq 50 \% \text { do } \\
\text { total }\end{array}$} & \multirow{3}{*}{$\begin{array}{l}14 \text { termos: Aparelho muito alto/Som muito alto, } \\
\text { Aparelho abafado/Som abafado, Aparelho com } \\
\text { eco/Som com eco/Eco na cabeça/Própria voz } \\
\text { com eco, Aparelho muito forte/Som muito forte, } \\
\text { Aparelho faz barulho/Muito barulho, Aparelho } \\
\text { chiando/Som chiado, Aparelho incomoda/ } \\
\text { Som incomoda/Incômodo, Dificuldade para } \\
\text { interpretação da fala, Aparelho fraco/Som fraco, } \\
\text { Som distorcido, Som cortando/Fala cortada, } \\
\text { Aparelho ruidoso/Ruído/Muito ruído, Própria voz } \\
\text { alta, Não ouve bem. }\end{array}$} & $\begin{array}{l}\text { Ajuste das características eletroacústicas: Aparelho } \\
\text { muito alto/Som muito alto, Aparelho abafado/Som } \\
\text { abafado, Aparelho muito forte/Som muito forte, } \\
\text { Aparelho faz barulho/Muito barulho, Aparelho } \\
\text { incomoda/Som incomoda/Incômodo, Aparelho fraco/ } \\
\text { Som fraco, Som distorcido, Som cortando/Fala cortada, } \\
\text { Aparelho ruidoso/Ruído/Muito ruído, Própria voz alta, } \\
\text { Não ouve bem. }\end{array}$ \\
\hline & & & $\begin{array}{l}\text { Adaptação física ou ajuste das características } \\
\text { eletroacústicas: Aparelho com eco/Som com eco/eco } \\
\text { na cabeça/Própria voz com eco, Aparelho chiando/Som } \\
\text { chiado (adaptação física quando referindo a feedback } \\
\text { acústico) }\end{array}$ \\
\hline & & & $\begin{array}{l}\text { Características intrínsecas do usuário: Dificuldade para } \\
\text { interpretação da fala. }\end{array}$ \\
\hline Categoria D & $\begin{array}{l}\text { Soma dos três } \\
\text { maiores valores } \leq \\
50 \% \text { do total }\end{array}$ & $\begin{array}{l}1 \text { termo: Escuto mas não entendo/Não consegue } \\
\text { entender nada/Não entende conversas. }\end{array}$ & $\begin{array}{l}\text { Características intrínsecas do usuário: Escuto mas não } \\
\text { entendo/Não consegue entender nada/Não entende } \\
\text { conversas. }\end{array}$ \\
\hline
\end{tabular}


enfrentam ${ }^{(23,24)}$. No entanto, evidências permanecem conflitantes quanto à confiabilidade da solução dos problemas baseada nas descrições e nos relatos dos próprios usuários ${ }^{(9,17,18,20,25)}$.

Tendo em vista que optou-se por uma análise dos termos e por uma separação buscando similaridade de terminologia, por meio da estrutura dos morfemas das palavras, ressalta-se que nos 30 termos há similaridade entre alguns quanto à queixa apresentada versus ajuste do AASI versus resultado para o paciente. Entende-se que existem termos ocorrentes, como "Aparelho muito alto/Som muito alto" (termo 1) e "Aparelho muito forte/Som muito forte" (termo 7), que, em uma análise classificatória do que a queixa representa, estão relacionados, podendo referir a mesma queixa. Desse modo, evidencia-se ainda mais que é uma queixa frequente e que o AASI está excedendo os níveis de conforto do paciente.

Dentro dessa ótica de análise, os termos "Aparelho baixo/ Som baixo" (termo 6), "Aparelho fraco/Som fraco" (termo 13), "Aparelho não faz diferença/Não faz diferença" (termo 19), "Não ouve bem" (termo 28) e "Não ouve nada" (termo 30), seguem a mesma linha de raciocínio quando se levanta uma possível solução: aumentar o ganho do AASI. Entende-se, no entanto, que somente o uso desses termos para descrever as queixas as tornam ainda inespecíficas, sendo necessária maior investigação para uma resolução precisa. As sugestões levantadas demonstram o passo inicial para guiar o fonoaudiólogo na realização de ajustes finos. Isso se torna ainda mais evidente quando se pensa na maneira que os AASI atuais processam o som e fornecem diversas transformações digitais no som que são encaminhadas para o usuário, na intenção de melhorar a inteligibilidade de fala ${ }^{(26)}$.

De acordo com a categorização proposta, observou-se que os termos "Aparelho sai" e "Aparelho escapa" foram diretamente relacionados à questão "se o molde ou oliva está muito largo", e o termo "Incômodo físico" relacionado com "se o molde ou oliva está muito apertado", ou seja, queixas referentes às características físicas do molde/oliva do AASI. Concordando com outro estudo, constatou-se que, muitas vezes, a dificuldade está relacionada ao manejo do AASI, o que pode ser considerado como fator de maior impacto na adesão ao processo de reabilitação auditiva, demonstrando que a orientação e o aconselhamento são um bom ponto de partida para auxiliar os usuários ${ }^{(23)}$.

No processo de adaptação de AASI, o primeiro passo é garantir a boa adaptação física: o AASI não pode se deslocar durante o uso, como demonstraram os termos "Aparelho sai" (termo 21) e "Aparelho escapa" (termo 29), o que já traduz que o AASI está mal adaptado. Mais adiante, os termos "Machuca (aparelho)" (termo 12), "Dor física/Dor na orelha" (termo 15) e "Incômodo físico" (termo 22) se referem ao fato de que, além do AASI estar mal posicionado, pode provocar um desconforto físico ao usuário. No entanto, o conforto físico é primordial para usar o AASI por longos períodos e obter os benefícios quanto à estimulação do sistema auditivo periférico e central. Visto que todas as queixas elencadas na categoria A estão diretamente relacionadas à adaptação física, o fonoaudiólogo deve direcionar seu olhar para esse aspecto, na tentativa de solucionar o problema apresentado.

Para a categoria $\mathrm{B}$, mesmo que os termos utilizados não estivessem integralmente relacionados a somente um comportamento do AASI, apresentaram a tendência de representar, mais de $50 \%$ das vezes, um único comportamento do dispositivo. Questões sobre adaptação física ficam mais fáceis de serem direcionadas e resolvidas quando utilizados os termos "Machuca (aparelho)",
"Ouvido tampado/Ouvido tampado (aparelho)", "Dor física/ Dor de orelha", referidos nessa categoria. Outros termos já podem elucidar a ideia da necessidade de ajustes finos, como os termos "Som estridente", "Aparelho estranho/Som estranho", "Aparelho não faz diferença/Não faz diferença", "Som forte incomoda", "Som metálico", "Não ouve nada". Os termos "Aparelho apitando" e "Própria voz dentro da cabeça" podem direcionar os fonoaudiólogos para dois caminhos: adaptação física ou ajuste das características eletroacústicas. Isso porque o "Aparelho apitando", referido pelo usuário, pode ser causado por mal posicionamento do AASI na orelha (características de adaptação física), ou por excesso de amplificação, ocasionando, assim, o feedback. Já o termo "Própria voz dentro da cabeça" leva a imaginar a presença de um efeito de oclusão, que deve ser resolvido com modificações na ventilação e profundidade da adaptação no meato acústico externo ou, em alguns casos, modificando o ganho de frequências baixas.

A maioria dos termos utilizados pelos usuários para se referirem a alguma queixa do AASI esteve relacionada a diversos comportamentos e características do dispositivo, como evidenciado na categoria C. Ao detalhar o olhar sobre essa categoria, verifica-se o aparecimento de uma situação problema com possíveis causas alternativas anteriormente referidas, as características intrínsecas do usuário. Já descrito na literatura, sabe-se que o AASI contribuiu como "porta de entrada" do som para o indivíduo. No entanto, a interpretação da mensagem e a compreensão da fala se dá no cérebro ${ }^{(27,28)}$. Dessa forma, muitas vezes, por mais que o fonoaudiólogo garanta acesso aos sons da fala e, por meio dos recursos fornecidos pelos AASI, diminua o esforço auditivo do usuário, a capacidade de acompanhar a conversação e entendimento da fala permanece comprometida, visto que aspectos cognitivos e cerebrais são essenciais para tal.

Ainda analisando a categoria $\mathrm{C}$, diversas queixas devem ser olhadas do ponto de vista da necessidade de refinamento da regulagem do AASI. Quando se observam queixas como "Aparelho muito alto/Som muito alto, "Aparelho muito forte/ Som muito forte", "Aparelho incomoda/Som incomoda/ Incômodo", o ajuste fino deve ser direcionado para questões de saída máxima e/ou ganho do AASI. Para queixas como "Aparelho faz barulho/Muito barulho", "Aparelho ruidoso/ Ruído/Muito ruído", sugere-se, também, verificar os recursos tecnológicos oferecidos pelo AASI, como os redutores de ruído.

A categoria $\mathrm{D}$ foi representada por um único termo: "Escuto mas não entendo/Não consegue entender nada/Não entende conversas". Essa é uma queixa que pode representar inúmeras situações que estão ocorrendo com o paciente e que deve levar o fonoaudiólogo a procurar um descritor mais preciso para realizar o ajuste fino do AASI. Assim como no estudo de Jenstad et al. ${ }^{(17)}$, para lidar com o problema dos termos imprecisos, é necessário questionar mais o paciente para encontrar um termo mais adequado que descreva e esclareça sua queixa.

Acredita-se que, independentemente do termo referido pelo usuário, é papel do fonoaudiólogo esmiuçar essa queixa e ser capaz de ser pontual, certeiro e específico na resolução do problema, respeitando as características do usuário ${ }^{(29)}$

Mesmo com os avanços e o uso das tecnologias digitais, é imprescindível o conhecimento do profissional a respeito das queixas referidas pelos usuários e de como trabalhá-las, assim como a necessidade de orientação sobre o AASI e o aconselhamento ao longo de todo o acompanhamento do usuário, o que concorda com o apontado em diferentes estudos ${ }^{(23,24,29,30)}$. Escutar e compreender o paciente traz uma adaptação efetiva, 
pois, de nada adianta o fonoaudiólogo possuir o treinamento técnico operacional sobre o AASI e sobre o software, e não saber compreender a queixa do paciente para transpor tal problema em forma de ajuste para sanar as queixas.

É necessário reforçar, também, que o processo de seleção e de adaptação do AASI compreende etapas de boas práticas que já são conhecidas e que guiam o fonoaudiólogo para um trabalho efetivo. Dentro dessa ótica, a verificação das características de amplificação de forma objetiva, por meio do uso do microfone sonda (considerado padrão-ouro na etapa de verificação) ${ }^{(7,8)}$, juntamente com a etapa de avaliação dos resultados com os testes de percepção de fala no silêncio e no ruído, que são capazes de avaliar como será o desempenho do paciente no seu dia a dia em sua comunicação usual ${ }^{(8)}$, somadas à parceria entre o indivíduo e o profissional ${ }^{(29)}$, em termos de relato de suas experiências e queixas, conduzem ao processo de adaptação de AASI com sucesso, permeado, sempre, de aconselhamento e orientações ao usuário.

Diante do exposto, mesmo com a limitação do estudo quanto à disparidade de respostas por fonoaudiólogos por região, 0 que pode não ser representativo de extensão territorial, foi possível conhecer as queixas mais recorrentes e conduzir um caminho para elas. Mais que isso, pôde-se reforçar a importância do papel do fonoaudiólogo durante o processo de seleção e adaptação do AASI.

\section{CONCLUSÃO}

Foi possível elencar as queixas mais referidas pelos usuários de AASI. Observou-se que é viável, a partir de uma queixa, direcionar um suposto aspecto relacionado à adaptação e auxiliar os fonoaudiólogos a levantar soluções, apesar de ser clara a necessidade de um olhar individualizado para cada paciente e situação.

Foram levantados 30 termos mais frequentes utilizados por usuários para queixas referentes ao seu AASI. O termo que mais apareceu foi "Aparelho muito alto/Som muito alto", com 223 ocorrências, e o que menos apareceu foi "Não ouve nada", com 25 ocorrências.

Dentro das categorias de análise dos termos previamente estabelecidas no intuito de estabelecer possíveis soluções de problemas, os termos foram relacionados aos aspectos principais do processo de adaptação desses dispositivos. Observou-se que, na categoria $\mathrm{A}$, as três queixas estavam diretamente relacionadas à adaptação física; na categoria $\mathrm{B}$, três queixas relacionadas à adaptação física, seis ao ajuste de características eletroacústicas e duas queixas que poderiam estar relacionadas a ambos os aspectos; na categoria $\mathrm{C}, 11$ queixas sugerindo necessidade de ajuste de características eletroacústicas, duas com a possibilidade de se referir à adaptação física ou ajuste de características eletroacústicas e uma com as características intrínsecas do usuário; na categoria $\mathrm{D}$, a queixa referida priorizou o direcionamento para características intrínsecas do usuário, quando se considera que o AASI está regulado da maneira adequada.

Entende-se que esses termos podem ser relacionados a determinados aspectos da adaptação do AASI. Mesmo um termo que possa identificar mais de uma queixa permite, a partir de uma análise conjunta, levantar possibilidades para os diferentes comportamentos e alcançar uma solução diferenciada para cada paciente.

\section{AGRADECIMENTOS}

Aos fonoaudiólogos que dedicaram o seu tempo para responder ao questionário e à UNICAMP, pela possibilidade de realização desta pesquisa.

\section{REFERÊNCIAS}

1. World Health Organization. Deafness and hearing loss [Internet] 2020 [citado em 2020 Abr 27]. Disponível em: https://www.who.int/ news-room/fact-sheets/detail/deafness-and-hearing-loss

2. Olusanya BO, Neumann KJ, Saunders JE. The global burden of disabling hearing impairment: a call to action. Bull World Health Organ. 2014;92(5):367-73. http://dx.doi.org/10.2471/BLT.13.128728. PMid:24839326.

3. Chisolm TH, Johnson CE, Danhauer JL, Portz LJP, Abrams HB, Lesner $\mathrm{S}$, et al. A systematic review of health-related quality of life hearing aids: final report of the American Academy of Audiology task force on the health-related quality of life benefits of amplication in adults. J Am Acad Audiol. 2007;18(2):151-83. http://dx.doi.org/10.3766/ jaaa.18.2.7. PMid:17402301.

4. Glick HA, Sharma A. Cortical neuroplasticity and cognitive function in early-stage, mild-moderate hearing loss: evidence of neurocognitive benefit from hearing aid use. Front Neurosci. 2020;14:93. http://dx.doi. org/10.3389/fnins.2020.00093. PMid:32132893.

5. Pichora-Fuller MK, Singh G. Effects of age on auditory and cognitive processing: implications for hearing aid fitting and audiologic rehabilitation. Trends Amplif. 2006;10(1):29-59. http://dx.doi. org/10.1177/108471380601000103.

6. Rodrigues CCC, Aurélio FS, Silva VB, Lopes TA. Benefício fornecido pelo uso de aparelhos de amplificação sonora individual em idosos de um programa de saude auditiva de Porto Velho - RO. Rev CEFAC. 2013;15(5):1170-80. http://dx.doi.org/10.1590/S151618462013005000025 .

7. Valente M, Abrams H, Benson D, Chisolm T, Citron D, Hampton $\mathrm{D}$, et al. Guidelines for the audiologic management of adult hearing impairment [Internet]. 2006 [citado em 2021 Jul 3]. Disponível em: https://audiology-web.s3.amazonaws.com/migrated/haguidelines. pdf_53994876e92e42.70908344.pdf

8. Almeida K, Mondelli MFCG. Boas práticas: caminho para uso com sucesso de próteses auditivas. In: $34^{\circ}$ Encontro Internacional de Audiologia - Fórum de Aparelhos de Amplificação Sonora Individual; 2019; Foz do Iguaçu. São Paulo: ABA; 2019. p. 1-7.

9. Anderson MC, Arehart KH, Souza PE. Survey of current practice in the fitting and fine-tuning of common signal-processing features in hearing aids for adults. J Am Acad Audiol. 2018;29(2):118-24. http:// dx.doi.org/10.3766/jaaa.16107. PMid:29401059.

10. Oh SH, Lee J. General framework of hearing aid fitting management. J Audiol Otol. 2016;20(1):1-7. http://dx.doi.org/10.7874/jao.2016.20.1.1. PMid:27144226.

11. Freiberger F. O acompanhamento fonoaudiológico de idosos usuários de aparelho de amplificação sonora individual: motivos da não aderência [dissertação]. Florianópolis: Universidade Federal de Santa Catarina; 2011. 129 p.

12. Gabrielsson A, Schenkman BN, Hagerman B. The effects of different frequency responses on sound quality judgment sand speech intelligibility. 
J Speech Hear Res. 1988;31(2):166-77. http://dx.doi.org/10.1044/ jshr.3102.166. PMid:3398490.

13. Gabrielsson A, Hagerman B, Bech-Kristensen T, Lundberg G. Perceived sound quality of reproductions with different frequency responses and sound levels. J Acoust Soc Am. 1990;88(3):1359-66. http://dx.doi. org/10.1121/1.399713. PMid:2229671.

14. Ovegård A, Lundberg G, Hagerman B, Gabrielsson A, Bengtsson $\mathrm{M}$, Brändström U. Sound quality judgement during acclimatization of hearing aid. Scand Audiol. 1997;26(1):43-51. http://dx.doi. org/10.3109/01050399709074974. PMid:9080554.

15. Gabrielsson A. Dimension analyses of perceived sound quality of sound-reproducing systems. Scand J Psychol. 1979;20(1):159-69. http://dx.doi.org/10.1111/j.1467-9450.1979.tb00697.x.

16. Moore BCJ, Alcántara JI, Glasberg BR. Development and evaluation of a procedure for fitting multi-channel compression hearing aids. $\mathrm{Br} \mathrm{J}$ Audiol. 1998;32(3):177-95. http://dx.doi.org/10.3109/03005364000000062. PMid:9710336.

17. Jenstad LM, Van Tasell DJ, Ewert C. Hearing aid troubleshooting based on patients' descriptions. J Am Acad Audiol. 2003;14(7):34760. http://dx.doi.org/10.1055/s-0040-1715754. PMid:14620609.

18. Thielemans T, Pans D, Chenault M, Anteunis L. Hearing aid fine-tuning based on Dutch descriptions. Int J Audiol. 2017;56(7):507-15. http:// dx.doi.org/10.1080/14992027.2017.1288302. PMid:28635499.

19. Bertozzo MC, Blasca WQ. Comparative analysis of the NAL-NL2 and DSL v5.0a prescription procedures in the adaptation of hearing aids in the elderly. CoDAS. 2019;31(4): e20180171. PMid:31433039.

20. Dreschler WA, Keidser G, Convery E, Dillon H. Client-based adjustments of hearing aid gain: the effect of different control configurations. Ear Hear. 2008;29(2):214-27. http://dx.doi.org/10.1097/ AUD.0b013e31816453a6. PMid:18490863.

21. Sabin AT, Gallun FJ, Souza PE. Acoustical correlates of performance on a dynamic range compression discrimination task. J Acoust Soc
Am [Internet]. 2013 Sep 26 [citado em 2021 Jul 3];134(3):2136-47. Disponível em: https://asa.scitation.org/doi/abs/10.1121/1.4816410

22. McShefferty D, Whitmer WM, Akeroyd MA. The just-noticeable difference in speech-to-noise ratio. Trends Hear. 2015 Jan;19:1-9. http://dx.doi.org/10.1177/2331216515572316. PMid:25681327.

23. Bennett RJ, Laplante-Lévesque A, Meyer CJ, Eikelboom RH. Exploring hearing aid problems: perspectives of hearing aid owners and clinicians. Ear Hear. 2018;39(1):172-87. http://dx.doi.org/10.1097/ AUD.0000000000000477. PMid:28787315.

24. McCormack A, Fortnum H. Why do people fitted with hearing aids not wear them? Int J Audiol. 2013;52(5):360-8. http://dx.doi.org/10. 3109/14992027.2013.769066. PMid:23473329.

25. Caswell-Midwinter B, Whitmer WM. The perceptual limitations of troubleshooting hearing-aids based on patients' descriptions. Int $\mathrm{J}$ Audiol. 2021;60(6):427-37. http://dx.doi.org/10.1080/14992027.20 20.1839679. PMid:33176515.

26. Nelson JA. Fine tuning multi-channel compression hearing instruments. Hear Rev. 2001;8(1):30-5.

27. Buchweitz A. Language and reading development in the brain today: neuromarkers and the case for prediction. J Pediatr. 2016 May;92(3):813. http://dx.doi.org/10.1016/j.jped.2016.01.005.

28. Benítez-Burraco A, Murphy E. Why brain oscillations are improving our understanding of language. Front Behav Neurosci. 2019;13:190. PMid:31551725.

29. Poost-Foroosh L, Jennings MB, Shaw L, Meston CN, Cheesman MF. Factors in client-clinician interaction that influence hearing aid adoption. Trends Amplif. 2011;15(3):127-39. http://dx.doi. org/10.1177/1084713811430217. PMid:22155784.

30. Bennett RJ, Meyer CJ, Eikelboom RH, Atlas JD, Atlas MD. Factors associated with self-reported hearing aid management skills and knowledge. Am J Audiol. 2018 Dez 6;27(4):604-13. http://dx.doi. org/10.1044/2018_AJA-18-0053. PMid:30286248. 